\title{
Single photons for all
}

\section{The observation of single-photon emission from defects in a two-dimensional semiconductor could lead to rapid developments in the pursuit of reliable and low-cost single-photon sources.}

A few weeks ago we published two papers reporting the observation of single-photon emission from defects in the plane of a layer of the two-dimensional semiconductor $\mathrm{WSe}_{2}$ (refs 1,2), and two more on the emission of single photons from defects formed at the interface between layers of $\mathrm{WSe}_{2}$ (refs 3,4). All of these papers are included in this issue.

Single-photon generation, which is essential for a range of applications in quantum communication, has been achieved with a variety of systems in the past, most notably with semiconductor quantum dots, atomic defects such as nitrogen-vacancy centres in diamond, and even organic molecules. Each system has its advantages, but also its limitations. It is, for example, still a challenge to find a single-photon source that is stable, can be replicated, and can be easily interfaced with electrical contacts - all desirable, if not essential, features for efficient quantum communication devices.

The results on $\mathrm{WSe}_{2}$ are promising on various fronts. The material is inorganic, and stable. It could be easily used to form heterostructures with other two-dimensional materials, such as graphene or boron nitride, allowing a single-photon emitter or detector to be embedded in a more complex device ${ }^{5}$. It is also relatively simple to place contacts on a WSe $e_{2}$ layer and apply an electrical voltage. Nick Vamivakas and colleagues show that, for instance, an applied voltage can affect the single-photon emission ${ }^{4}$.

As Vasili Perebeinos explains in his News and Views article ${ }^{6}$, the observation of single-photon emission is only the first step towards the realization of quantum communication devices based on a twodimensional semiconductor. It will be important to understand the origin of the defects and how to create them in a controlled way. Coupling the defects with a photonic cavity may enhance the optical emission and, especially, increase the speed at which photons are emitted. It may be possible to obtain single photons from the recombination of electrons and holes injected through metallic contacts.
For use in realistic devices it will be essential to design structures that can provide single-photon emission at room temperature. Clearly there is still a lot to do. The good news is that two-dimensional semiconductors can be isolated relatively easily, at least by mechanical exfoliation, which is perfectly adequate for fundamental studies. This is undoubtedly one of the reasons why a number of research groups were able to observe single-photon emission almost at the same time. Apart from the papers in this issue ${ }^{1-4}$, at least another one (to our knowledge) has already been published elsewhere ${ }^{7}$. There is, therefore, reason to be optimistic and expect that developments will happen rapidly.

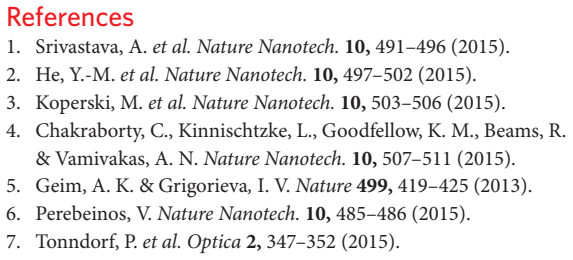

\section{Notes on a retraction}

\section{Correcting the scientific literature with the help of the research community.}

On 5 May 2013, Nature Nanotechnology published a research article 'DNA sequencing using electrical conductance measurements of a DNA polymerase' by G. Steven Huang and colleagues (Nature Nanotech. 8, 452-458; 2013). The work suggested that the sequence of bases in single molecules of DNA could be identified by monitoring the electrical conductance of a DNA polymerase as it incorporated unlabelled nucleotides into the DNA strand. We are now retracting this paper (Nature Nanotech. 10, 563; 2015).

Shortly after publication, concerns were raised about the validity of the data by a number of researchers in the community. After evaluating the concerns carefully, we contacted the authors' institution, the National Chiao Tung University, and asked them to launch a formal investigation into the case. An editorial note about the paper was also published in the journal at this time (Nature Nanotech. 8, 781; 2013). The university focused their investigation on the reproducibility of the data. The authors could not reproduce the results of the work within the timeframe set by the investigating committee, and could not provide the committee with a complete set of raw data for the original experiments. In light of this, the authors agreed to retract the paper.

The retraction serves to correct the scientific record, and in writing this editorial we wanted to acknowledge the efforts of those researchers who voiced their scientific concerns and made this possible. They were, in particular, Vincent Croquette of the Ecole Normale Supérieure in Paris; Stuart Lindsay of Arizona State University; Philip Collins of the University of California, Irvine; Marija Drndic of the University of Pennsylvania; Jens Gundlach of the University of Washington; Brett Gyarfas of Arizona State University; Meni Wanunu of Northeastern University; Mark Akeson of the University of California, Santa Cruz; Kate Lieberman of the University of California, Santa Cruz; and Stephen Turner of Pacific Biosciences. All of the researchers named here have chosen not to remain anonymous.

Pre-publication peer review is a vital component of our publication process, providing both a technical assessment of a piece of work and an evaluation of its wider significance. In cases where issues do arise after publication, we are indebted to the researchers who choose to get involved. Indeed, it is the efforts of the research community that enable our peer review, and it is the efforts of the same research community that help us to correct the publication record when that is needed. The retraction is a clear reminder that the process of evaluating a paper does not end when the work is published. 